\title{
PERÍODO DE INDUÇÃO DE BLENDAS DE BIODIESEL DE DIFERENTES FONTES GRAXAS
}

\author{
J. G. Teleken ${ }^{143 *}$ \\ P. A. Cremonez ${ }^{144}$ \\ M. Feroldi ${ }^{145}$ \\ C. De J. De Oliveira ${ }^{146}$ \\ T. R. W. Meier ${ }^{147}$ \\ J. Dieter ${ }^{148}$ \\ E. I. B. Parisotto ${ }^{149}$
}

\begin{abstract}
RESUMO
O presente trabalho teve como objetivo avaliar o efeito da estabilidade a oxidação do biodiesel de soja pela blendagem com outros biodieseis advindos de diferentes fontes graxas amplamente. Para condução do experimento realizou-se planejamento fatorial ao nível $2^{3}$ misturando-se biodieseis de soja em diferentes níveis com biodiesel de milho, pequi e gordura suína, todos estes obtidos via transesterificação homogênea alcalina, para avaliação da estabilidade oxidativa (período de indução). A partir dos resultados obtidos pode-se concluir que a blendagem de biodieseis de diferentes origens proporciona variações nas respostas de estabilidade à oxidação do combustível. Enquanto a adição de ésteres advindos de fonte vegetal garantiu melhora na estabilidade oxidativa, ésteres de gordura suína proporcionaram declínio nas respostas obtidas a partir de sua blendagem com o biodiesel de soja.
\end{abstract}

PALAVRAS-CHAVES: período de indução; culturas energéticas; rancimat.

\section{INTRODUÇÃO}

\footnotetext{
${ }^{143}$ Federal University of Paraná, Department Engineering and Exacts, R. Pioneiro, 2153, CEP: 85.950000, Bairro Jardim Dallas, Palotina - PR, Brazil.

${ }^{*}$ Corresponding author: e-mail: joelteleken@gmail.com. Phone: +55(44)32118586

144 University of West Paraná, Paraná, Brazil. Department of Agricultural Energy. Rua Universitária, 2069, CEP: 85.819-130, Bairro Faculdade, Cascavel - PR, Brazil.

145 University of West Paraná, Paraná, Brazil. Department of Agricultural Energy. Rua Universitária, 2069, CEP: 85.819-130, Bairro Faculdade, Cascavel - PR, Brazil.

${ }^{146}$ Federal University of Paraná, Department Engineering and Exacts, R. Pioneiro, 2153, CEP: 85.950000, Bairro Jardim Dallas, Palotina - PR, Brazil.

${ }^{147}$ Federal University of Paraná, Department Engineering and Exacts, R. Pioneiro, 2153, CEP: 85.950000, Bairro Jardim Dallas, Palotina - PR, Brazil.

${ }^{148}$ Federal University of Paraná, Department Engineering and Exacts, R. Pioneiro, 2153, CEP: 85.950000, Bairro Jardim Dallas, Palotina - PR, Brazil.

149 Federal University of Santa Catarina, Department Engineering Chemistry and Food, Florianópolis, Santa Catarina, Brazil.
} 
O biodiesel é visto como um combustível renovável natural que apresenta potencial poluidor inferior ao diesel convencional e pode ser utilizado como alternativa a esse combustível fóssil (CANDEIA et al. 2009; DINKOV et al. 2009), sendo que as principais matérias-primas utilizadas para sua obtenção são óleos e gorduras vegetais e animais.

Apesar das diversas vantagens do biodiesel, por sua característica biodegradável, este combustível é mais susceptível a auto-oxidação se comparado aos combustíveis fósseis (CANDEIA et al. 2009).

A estabilidade oxidativa do biodiesel é afetada por inúmeros fatores durante seus processos de transporte, armazenamento e comercialização (DANTAS et al. 2011). A auto oxidação ocorre pela reação em cadeia de radical livre entre o combustível e o oxigênio presente na atmosfera, produzindo peróxidos, hidroperóxidos, ácidos carboxílicos, etc (VIEIRA; PASQUINI, 2014).

Dentre os problemas decorrentes da alta degradação oxidativa do biodiesel, destaca-se o aumento da viscosidade, elevação da acidez e formação de gomas, além de outros compostos indesejáveis (TANG et al. 2008). Os produtos de oxidação ainda são corrosivos para as câmaras dos motores e podem ocasionar o entupimento de bombas de injeção e filtros (DANTAS et al. 2011).

A determinação da estabilidade oxidativa do biodiesel pelo método Rancimat (método oficial da norma européia EM 14112 para biodiesel) é obtida pela determinação do tempo de indução da amostra (TI), baseado no aumento da condutividade da água que retém os ácidos voláteis liberados durante a oxidação do combustível. Este método exige período de indução mínimo de 6 horas para o biodiesel (FAME, 2003; WAZILEWSKI et al., 2013).

Frente a este contexto, o presente trabalho teve como objetivo avaliar o efeito do período de indução do biodiesel de soja pela blendagem com outros biodieseis advindos de diferentes fontes graxas amplamente encontradas no Brasil.

\section{MATERIAIS E MÉTODOS}

Para desenvolvimento do atual estudo utilizou-se óleo de soja (S), além de óleo de milho $(M)$, óleo bruto de pequi $(P)$ e de gordura suína $(G)$. Todas as matérias-primas foram acondicionadas sob refrigeração até momento de utilização.

R. gest. sust. ambient., Florianópolis, n. esp, p.532-541, dez. 2015. 


\subsection{Transesterificação}

Empregaram-se $70 \mathrm{~g}$ de óleos ou gordura residuais de fritura na reação de transesterificação metanólica homogênea básica por 30 minutos sob agitação constante e temperatura de $55^{\circ} \mathrm{C}\left( \pm 2{ }^{\circ} \mathrm{C}\right)$. A relação molar álcool/material graxo foi fixada em excesso de 6:1, empregando $1 \%(\mathrm{~m} / \mathrm{m})$ de catalisador $\mathrm{NaOH}$ com relação à massa do material graxo (Liang et al., 2009; Kumar et al., 2013; e Miladinović et al. 2013).

Realizaram-se triplicatas para as reações de transesterificação com todos os materiais graxos. A mistura reacional foi centrifugada a 3000 RPM durante $10 \mathrm{~min}$ para separação das fases. Após, lavou-se a mistura de ésteres com água destilada a $55{ }^{\circ} \mathrm{C}$ durante $10 \mathrm{~min}$, e então centrifugou-se novamente para remoção da fase inferior, composta por sabão, parte do álcool em excesso e água. Então, a mistura foi levada a aquecimento $\left(100 \pm 5^{\circ} \mathrm{C}\right)$ durante $10 \mathrm{~min}$ para remoção da umidade e do álcool em excesso ainda presentes (adaptado de GERIS et al., 2007).

\subsection{Blendas}

Após a transesterificação dos materiais graxos, as misturas de ésteres foram blendadas respeitando a relação exposta na Tabela 1. Na Tabela 2 estão descritos os ensaios, segundo as proporções de blendagem dos ésteres produzidos pelos óleos residuais.

Tabela 1. Planejamento fatorial $2^{3}$

\begin{tabular}{llll}
\hline Tratamento & Pequi & Milho & Gordura \\
\hline 1 & - & - & - \\
5 & + & - & - \\
6 & - & + & - \\
7 & - & - & + \\
8 & + & + & - \\
9 & + & - & + \\
10 & - & + & + \\
11 & + & + & + \\
\hline
\end{tabular}

Fonte: Elaborada pelos autores, 2015.

Tabela 2. Proporções utilizadas na blendagem dos ésteres produzidos

\begin{tabular}{ccc}
\hline Tratamento & Blenda & Proporção \\
\hline 1 & S & 10 \\
2 & M & 10 \\
3 & G & 10 \\
4 & G & 10
\end{tabular}

R. gest. sust. ambient., Florianópolis, n. esp, p.532-541, dez. 2015. 


\begin{tabular}{ccc}
5 & SP & $8: 2$ \\
6 & SM & $8: 2$ \\
7 & SG & $8: 2$ \\
8 & SPM & $6: 2: 2$ \\
9 & SPG & $6: 2: 2$ \\
10 & SMG & $6: 2: 2$ \\
11 & SPMG & $4: 2: 2: 2$ \\
12 & SPMG & $7: 1: 1: 1$ \\
\hline
\end{tabular}

Fonte: Elaborada pelos autores, 2015

\subsection{Conversão de ésteres}

Para determinação da conversão do óleo empregado na pesquisa em ésteres alquílicos, utilizou-se de Cromatografia a gás com cromatógrafo Perkin Elmer (Clarus 680), equipado com um detector de ionização de chama (FID). Temperatura do forno iniciando em $160^{\circ} \mathrm{C}$, até $230^{\circ} \mathrm{C}$, a uma taxa de $5^{\circ} \mathrm{C} \mathrm{min}-1$, durante 6 minutos. Volume de injeção da amostra de $2 \mu \mathrm{L}$, no modo split 1:50. A vazão do gás de arraste foi de $1,5 \mathrm{~mL} \cdot \mathrm{min}^{-1}$. Utilizou-se uma coluna capilar (Perkin Elmer - Elite Wax, $30 \mathrm{~m} \times 0,25$ $\mathrm{mm} \times 0,5 \mu \mathrm{m})$.

\subsection{Estabilidade oxidativa}

A análise foi desenvolvida com uma taxa de insuflação de ar de 10 Lh-1 para determinar o período de indução, que precede a etapa de propagação em reações em cadeia radicais. $O$ teste foi realizado utilizando o equipamento Rancimat modelo 873 (Metrohm $®$ - Herisau/Switzerland) em conformidade com o padrão oficial para determinação da estabilidade oxidativa em teste acelerado, EN 14112.20.

\section{RESULTADOS E DISCUSSÃO}

Todos os materiais graxos transesterificados apresentaram valores de conversão em ésteres elevados, sendo de $90,10 \%, 97,13 \%, 86,66 \%$ e $91,88 \%$ para os óleos de soja, pequi, milho e gordura suína, respectivamente.

Os resultados obtidos para os períodos de indução dos biodieseis in natura e as blendas podem ser visualizados a partir da Tabela 3.

Tabela 3. Período de indução, índice de acidez e peróxido obtido em análise dos biodieseis puros e blendados.

R. gest. sust. ambient., Florianópolis, n. esp, p.532-541, dez. 2015. 


\begin{tabular}{cccc}
\hline Trat. & Blenda & Proporção & Período de Indução (h) \\
\hline 1 & S & 10 & 5,53 \\
2 & P & 10 & 6,18 \\
3 & M & 10 & 6,20 \\
4 & G & 10 & 1,15 \\
5 & SP & $8: 2$ & 5,63 \\
6 & SM & $8: 2$ & 5,94 \\
7 & SG & $8: 2$ & 2,24 \\
8 & SPM & $6: 2: 2$ & 5,83 \\
9 & SPG & $6: 2: 2$ & 3,30 \\
10 & SMG & $6: 2: 2$ & 5,43 \\
11 & SPMG & $4: 2: 2: 2$ & 3,99 \\
12 & SPMG Central & $7: 1: 1: 1$ & $4,75( \pm 0,11)$ \\
\hline
\end{tabular}

Fonte: Elaborada pelos autores, 2015

A norma européia EN 14214 utilizada para determinação da estabilidade oxidativa a $110{ }^{\circ} \mathrm{C}$ estabelece um tempo mínimo de indução de 6 horas pelo método Rancimat enquanto que a norma americana ASTM D6751 determina período mínimo de 3 horas (KNOTHE, 2007; PULLEN; SAEED, 2012). Apenas os biodieseis puros de pequi e milho (Figura 1) apresentaram período de indução superior ao estabelecido pela norma européia, sendo que apesar da mistura proporcionar melhores resultados, nenhuma das blendas preparadas com óleo de soja apresentou estabilidade requerida.

No entanto, com relação ao limite estabelecido pela norma americana, apenas o biodiesel (B100) de gordura suína apresentou período de indução inferior ao estabelecido. Os baixos valores são decorrentes dos prolongados processos de cocção que propiciaram o aumento da acidez pela liberação de ácidos graxos livres e promoveram a aceleração da oxidação do material.

Apenas as misturas de biodiesel de soja com biodieseis de milho e gordura suína proporcionaram alterações significativas na variável estabilidade oxidativa através do período de indução. A superfície de resposta gerada para a mistura destes biodieseis pode ser visualizada a partir da Figura 2. O biodiesel de milho promoveu incremento significativo no período de indução do biodiesel de soja. Enquanto isso, o biodiesel de gordura suína promoveu um decréscimo considerável no período de indução do biodiesel aditivado. A mistura dos dois biodieseis junto aos ésteres de soja gera um efeito positivo no período de indução.

Os óleos vegetais apresentam em sua composição anti-oxidantes naturais, como os tocoferóis que aumentam a estabilidade do biodiesel. Por outro lado, as gorduras animais não possuem estes anti-oxidantes, sendo que o biodiesel produzido

R. gest. sust. ambient., Florianópolis, n. esp, p.532-541, dez. 2015. 
a partir destas fontes normalmente apresenta período de indução inferior ao dos biodieseis advindos de óleos vegetais (SENDZIKIENE et al. 2005; CUNHA Jr. et al. 2013).

Figura 1. Período de indução dos biodieseis de milho (direita) e pequi (esquerda).
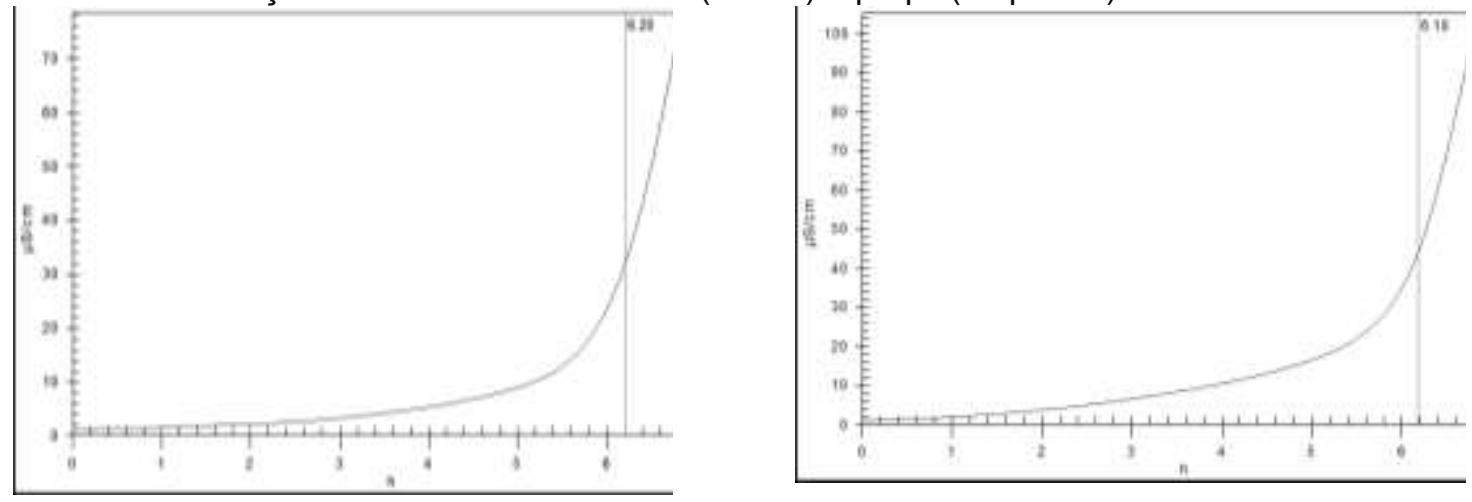

Fonte: Elaborada pelos autores, 2015

Figura 2. Superfície de resposta para adição de milho e gordura suína no período de indução do biodiesel de soja. Equação: IP=4,74+0,56M*-0,10G* $+0,41 \mathrm{MG}^{*}$. $F$ calculado: $16,8259 . R^{2}=0,878$. *correspondem aos termos que apresentaram significância ao nível de $5 \%$.

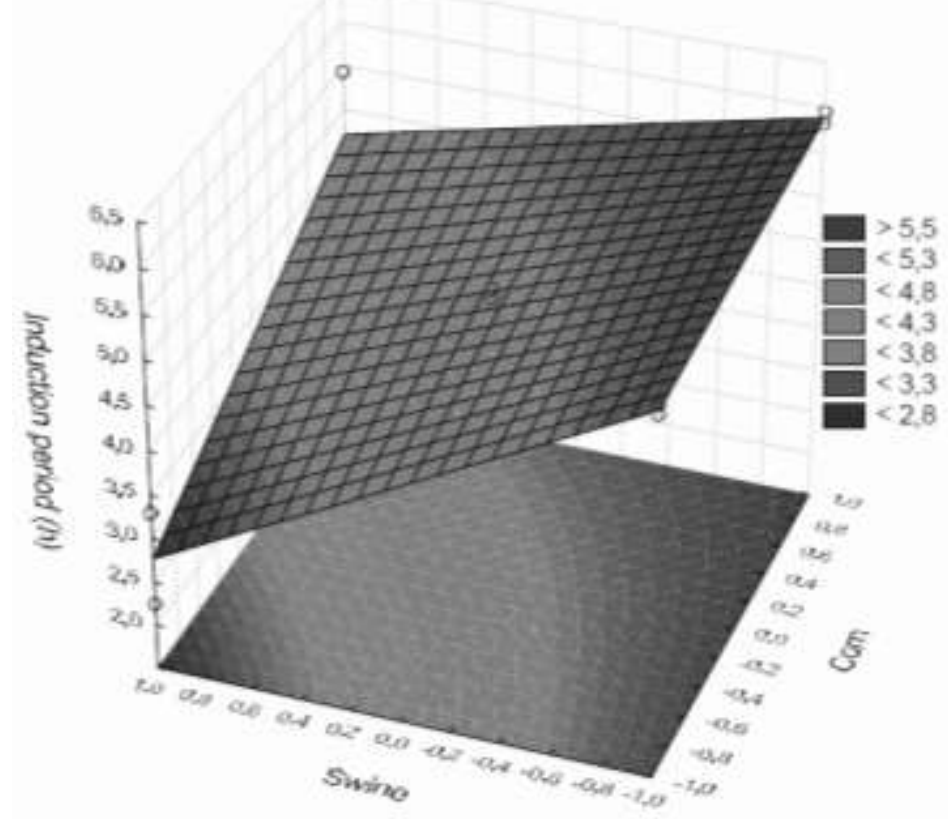

Fonte: Elaborada pelos autores, 2015

Apesar de sua característica vegetal, em decorrência de sua alta composição em ácidos poli-insaturados, o óleo de soja tem uma reatividade oxidativa alta que Ihe confere períodos de indução inferiores aos estabelecidos pelas normas européias e impedindo o armazenamento prolongado de seu biodiesel (MITTELBACH; GANGL, 2001; KINNEY; CLEMENTE, 2005). Pereira et al. (2015) obtiveram período de indução

R. gest. sust. ambient., Florianópolis, n. esp, p.532-541, dez. 2015. 
de 5,5 horas para o biodiesel de soja. Além deste, Sousa et al. (2014), constataram em pesquisa com biodiesel de soja período de indução de 4,97 horas, valores inferiores ao estabelecido pelos padrões europeus assim como o observado no presente estudo.

A oxidação do combustível gera borras e promove entupimento de filtros e mangueiras de combustível. Em decorrência disso, a necessidade de aditivos ou blendagens que garantam uma melhora da estabilidade oxidativa deste material graxo tem sido estudada.

Valores obtidos por Cunha Jr. et al. (2013) utilizando gordura suína também foram inferiores aos estabelecidos pelas normas, assim como o trabalho de Sendzikiene et al. (2005). Este fator inviabiliza a utilização deste material graxo como aditivo para melhoria da estabilidade oxidativa do biodiesel de soja. Resultados comparados em pesquisa de Cunha Jr et al. (2013), também mostram períodos de indução baixos (1,7 e 2,6 horas) necessitando a utilização de antioxidantes para adequação.

Galvan et al. (2014) blendando biodiesel de soja e de gordura suína em proporção de 9:1 obtiveram períodos de indução relativamente baixo alcançando 1,42 horas. Nadaleti et al. (2014) realizando mistura destes biodieseis nas mesmas proporções obtiveram período de indução de 3,61 horas.

Períodos de indução muito distintos são encontrados para o óleo e biodiesel de milho. Dantas et al. (2011), obtiveram para o óleo de milho período de indução de 2,89 horas, valor inferior ao obtido na atual pesquisa para o biodiesel utilizado. Já o trabalho de Lima et al. (2010) gerou biodiesel que apresentou período de indução de 7,75 horas enquanto seu óleo apresentou valor de 10,23 horas. Serqueira et al. (2014) obtiveram para o biodiesel de milho estabilidade oxidativa de 6,5 horas. Características como o elevado índice de acidez podem ser relevantes para obtenção de resultados discretos ou discrepantes.

Os valores de período de indução obtidos para o biodiesel de pequi produzido a partir de óleo in natura variou entre 4,5 e 4,95 horas em pesquisa de Silva et al. (2014).

Ainda que o biodiesel de milho possa ser utilizado para elevar o período de indução do biodiesel, este não garantiu que o biodiesel de soja alcançasse os valores estipulados pela norma européia, sendo necessário o emprego de antioxidantes para obtenção de resultados aceitáveis.

R. gest. sust. ambient., Florianópolis, n. esp, p.532-541, dez. 2015. 


\title{
4 CONSIDERAÇÕES FINAIS
}

A partir dos resultados obtidos pode-se concluir que a blendagem de biodieseis de diferentes origens proporciona variações nas respostas de estabilidade à oxidação do combustível. Enquanto a adição de ésteres advindos de fonte vegetal, rico em antioxidantes naturais como o milho, garantiu melhora na estabilidade oxidativa, ésteres de gordura suína proporcionou um declínio nas respostas obtidas a partir de sua blendagem com o biodiesel de soja.

\section{INDUCTION PERIOD BLENDS OF DIFERENT SOURCES BIODIESEL GREASE}

\begin{abstract}
This study aimed to evaluate the effect of oxidation stability of soybean biodiesel for blending with other biodiesels coming from different sources widely greases. To conduct the experiment took place at the level factorial design $2^{3}$ blending soybean biodiesels at different levels with corn, pequi and swine fat biodiesel, all these obtained at alkaline homogeneous transesterification, for evaluation of the oxidative stability (induction period). From the results obtained it can be concluded that the blending of biodiesels from different sources provides variations in the fuel oxidation stability responses. While the addition of esters deriving from vegetable source ensured improvement in the oxidative stability of swine fat esters provided decline in responses obtained from their blending with soybean biodiesel.
\end{abstract}

KEYWORDS: induction period; energy crops; rancimat.

\section{REFERÊNCIAS}

Candeia RA, da Silva MCD, Carvalho Filho JR, Brasilino MGA, Bicudo TC, Santos MG, et al., 2009. Influence of soybean biodiesel content on basic properties of biodiesel-diesel blends. Fuel 88, 738-43.

Cunha Jr. A, Feddern V, De Prá MC, Higarashi MM, Abreu PG, Coldebella A., 2013. Synthesis and characterization of ethylic biodiesel from animal fat wastes. Fuel 105, 228-234.

Dantas MB, Albuquerque AR, Barros AK, Rodrigues Filho MG, Antonisio Filho NR, et al., 2011. Evaluation of the oxidative stability of corn biodiesel. Fuel 90, 773-778.

Dinkov R, Hristov G, Stratiev D, Aldary VB., 2009. Effect of commercially available antioxidants over biodiesel/diesel blends stability. Fuel 88, 732-7.

R. gest. sust. ambient., Florianópolis, n. esp, p.532-541, dez. 2015. 
European Committee for Standardization, fatty acid methyl esters (FAME), 2013.

Determination of oxidation stability (accelerated oxidation test). Method EN14112.

Galvan D, Orives JR, Coppo RL, Rodrigues CHF, Spacino KR, 2014. Study of oxidation kinetics of b100 biodiesel from soybean and pig fat: activation energy determination. Quím. Nova 37(2), 244-248.

Geris R, Santos NAC dos, Amaral BA, et al., 2007. Biodiesel de soja - reação de transesterificação para aulas práticas de química orgânica. Quím. Nov. 30(5), 13691373.

Kinney AJ, Clemente TE., 2005. Modifying soybean oil for enhanced performance in biodiesel blends. Fuel Processing Technology 86, 1137-47.

Knothe G., 2007. Some aspects of biodiesel oxidative stability. Fuel Processing Technology 88(7), 669-677.

Kumar R, Tiwari P, Garg S., 2013. Alkali transesterification of linseed oil for biodiesel production. Fuel 104, 553-560.

Liang YC, May CY, Foon CS, Ngan MA, Hock CC, Basiron Y., 2006. The effect of natural and synthetic antioxidants on the stability of palm diesel. Fuel 85, 867-870.

Lima AL, Lima AP, Portela FM, Santos DQ, Neto WB, Hernández-Terrones MG, Fabris JD., 2010. Parâmetros da reação de transesterificação etílica com óleo de milho para produção de biodiesel. Eclética Química 35(4), 101-106.

Miladinović MR, Krstić JB, Tasić MB, et al., 2013. A kinetic study of quicklimecatalyzed sunflower oil methanolysis. Chem. Engin. Res. and Design. DOI: 10.1016/j.cherd.2013.11.023.

Mittelbach M, Gangl S., 2001. Long storgae stability of biodiesel made from rapeseed and used frying oil, J. Am. Oil Chem. Soc. 78, 573-577.

Nadaleti WC, Bariccatti RA, Alves HJ, de Souza SNM, Antonelli J, Cremonez PA, Belli Filho P, Mari Jr A., 2014. Factorial planning and surface response methodology in oxidative stability evaluation of biodiesel blends from soybean, crambe, babassu and swine fat. J. of Food, Agric. \& Environ. 12(2), 1030-1032.

Pereira GG, Morales A, Marmesat S, Ruiz-Méndez MV, Barrera-Arellano D, Dobarganes MC., 2015. Effect of temperature on the oxidation of soybean biodiesel. Grasas y Aceites 66(2), 72.

Pullen J, Saeed K., 2012. An overview of biodiesel oxidation stability. Renewable and Sustainable Energy Reviews 16(8), 5924-50.

Sendzikiene E, Makareviciene V, Janulis P., 2005. Oxidation stability of biodiesel fuel produced from fatty wastes. Pol J Environ Stud. 14(3), 335-9.

R. gest. sust. ambient., Florianópolis, n. esp, p.532-541, dez. 2015. 
Serqueira DS, Fernandes DM, Cunha RR, Squissato AL, Santos DQ, Richter EM, Munoz RAA. 2014. Influence of blending soybean, sunflower, colza, corn, cottonseed, and residual cooking oil methyl biodiesels on the oxidation stability. Fuel $118,16-20$.

Silva TA, de Assunção MN, Vieira AT, de Oliveira MF, Batista ACF. 2014. Methylic and ethylic biodiesels from pequi oil (Caryocar brasiliense Camb.): Production and thermogravimetric studies. Fuel, 136, 10-18.

Sousa LS, de Moura CVR, de Oliveira JE, de Moura EM. 2014. Use of natural antioxidants in soybean biodiesel. Fuel 134, 420-428.

Tang H, Wang A, Salley SO, Simon KY., 2008. The Effect of natural and synthetic antioxidants on the oxidative stability of biodiesel. J. Am. Oil Chem. Soc. 85, 373382.

Vieira FS, Pasquini C., 2014. Determination of the oxidative stability of biodiesel using near infrared emission spectroscopy. Fuel 117, 1004-1009.

Wazilewski WT, Bariccatti RA, Martins GI, Secco D, de Souza SNM, et al., 2013. Study of the methyl crambe (Crambe abyssinica Hochst) and soybean biodiesel oxidative stability. Industrial Crops and Products 43, 207-212.

R. gest. sust. ambient., Florianópolis, n. esp, p.532-541, dez. 2015. 